\title{
Computer simulation of coercivity improvement due to microstructural refinement
}

\author{
Hirotoshi Fukunaga, ${ }^{1, a)}$ Ryousuke Hori, ${ }^{1}$ Masaki Nakano, ${ }^{1}$ Takeshi Yanai, ${ }^{1}$ Ryutaro Kato, ${ }^{2}$ \\ and Yoshiyuki Nakazawa ${ }^{2}$ \\ ${ }^{1}$ Graduate School of Engineering, Nagasaki University, 1-14 Bunkyou-machi, Nagasaki 852-8521, Japan \\ ${ }^{2}$ Honda R\&D Co., Ltd., 4630 Shimotakanezawa, Tochigi 321-3393, Japan
}

(Presented 4 November 2014; received 22 September 2014; accepted 9 November 2014; published online 24 March 2015)

\begin{abstract}
The effect of the presence of a non-magnetic phase at multi-junctions of $\mathrm{Nd}_{2} \mathrm{Fe}_{14} \mathrm{~B}$ grains on the demagnetization process of $\mathrm{Nd}-\mathrm{Fe}-\mathrm{B}$ magnets was investigated by micromagnetic simulations, while varying the size of the non-magnetic phase and the $\mathrm{Nd}_{2} \mathrm{Fe}_{14} \mathrm{~B}$ grains. While the demagnetizing field created by the non-magnetic phase assisted the nucleation of a reverse domain, its effect was only significant when its size exceeded a critical value. This critical size effect can be explained by a change in the spatial distribution of the demagnetizing field. In addition, the calculations indicated that microstructural refinement would also increase the coercivity. This increase in coercivity could also be attributed to a change in the spatial distribution of the demagnetizing field due to the presence of a non-magnetic phase. Changes in the spatial distribution of the demagnetizing field due to such a non-magnetic phase could represent a mechanism by which microstructural refinement leads to increased coercivity. (C) 2015 AIP Publishing LLC.
\end{abstract}

[http://dx.doi.org/10.1063/1.4915905]

\section{INTRODUCTION}

An increase in the coercivity of $\mathrm{Nd}-\mathrm{Fe}-\mathrm{B}$ magnets is needed to enable their application to electric machines used at high temperatures such as electric and hybrid vehicles, because their coercivity is much lower than their anisotropy field and decreases drastically at high temperatures. ${ }^{1}$ Therefore, clarification of the origins of low coercivity is an important issue for the further improvement of coercivity. For example, a reduced ${ }^{2-4}$ or negative $^{5}$ magnetic anisotropy constant at grain surfaces, the existence of ferromagnetic grain boundaries, ${ }^{6}$ and a strong local demagnetizing field ${ }^{7}$ have been discussed as candidate origins. It has also been reported that coercivity increases with decreasing grain size. ${ }^{8,9}$ These observations and calculations suggest that the microstructure plays an important role in the determination of coercivity.

Micromagnetic studies have examined the effects of non-magnetic grain boundary phases, ${ }^{10-12}$ grain size, ${ }^{13-16}$ and grain surface defects. ${ }^{2,3,17}$ A collective effect of large non-magnetic phases located at the corners of $\mathrm{Nd}_{2} \mathrm{Fe}_{14} \mathrm{~B}$ grains has also been reported for a sintered magnet by computer simulations based on micromagnetic theory. ${ }^{18}$ However, these micromagnetic simulations have not clearly determined the effects of the size of the non-magnetic phases.

Therefore, in this investigation, the effect of the presence of a non-magnetic phase at multi-junctions of $\mathrm{Nd}_{2} \mathrm{Fe}_{14} \mathrm{~B}$ grains on the coercivity was studied by varying the sizes of the non-magnetic phase and the $\mathrm{Nd}_{2} \mathrm{Fe}_{14} \mathrm{~B}$ grains. It was

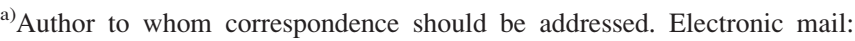
fukunaga@nagasaki-u.ac.jp.
}

found that the non-magnetic phase decreased the nucleation field remarkably when its size exceeded a critical value. It was also found that microstructural refinement increased the coercivity, which could be attributed to the critical size effect mentioned above.

\section{SIMULATION MODEL AND METHOD}

A cubic model with a side length $L$ was assumed, as shown in Fig. 1. The model is composed of a $\mathrm{Nd}_{2} \mathrm{Fe}_{14} \mathrm{~B}$ grain, a tetrahedral non-magnetic phase in contact with four $\mathrm{Nd}_{2} \mathrm{Fe}_{14} \mathrm{~B}$ grains, and non-magnetic grain boundaries between two adjacent $\mathrm{Nd}_{2} \mathrm{Fe}_{14} \mathrm{~B}$ grains. The value of $L$ was 48,96 , or $192 \mathrm{~nm}$, and the non-magnetic phase size $T$ was varied over the range from 0 to $0.75 \times L$. The thickness of the grain boundaries was $L / 32$. It was also assumed that the thin surface layers of the $\mathrm{Nd}_{2} \mathrm{Fe}_{14} \mathrm{~B}$ grain, which were $3 \mathrm{~nm}$ thick, had atomic defects and/or disorder, and the magnetic anisotropy constant for these layers, $K_{\mathrm{ud}}$, was smaller than that for the grain interior, $K_{\mathrm{u} \cdot}{ }^{4}$ An external field was applied along

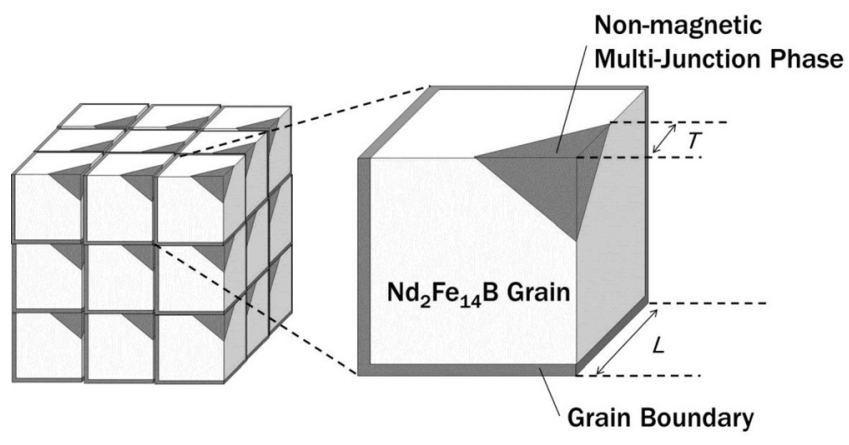

FIG. 1. Calculation model. 
TABLE I. Material parameters used in simulation.

\begin{tabular}{lccc}
\hline \hline & $\mathrm{Nd}_{2} \mathrm{Fe}_{14} \mathrm{~B}$ & $\begin{array}{c}\text { Surface } \\
\text { layer }\end{array}$ & $\begin{array}{c}\text { Non-magnetic } \\
\text { phase }\end{array}$ \\
\hline Anisotropic constant $K_{u}\left[\mathrm{MJ} / \mathrm{m}^{3}\right]$ & 4.5 & 2.25 or 4.5 & 0 \\
Saturation polarization $J_{s}[\mathrm{~T}]$ & 1.61 & 1.61 & 0 \\
Exchange stiffness constant $A[\mathrm{~J} / \mathrm{m}]$ & $8.7 \times 10^{-12}$ & $8.7 \times 10^{-12}$ & 0 \\
\hline \hline
\end{tabular}

the easy magnetization direction of the $\mathrm{Nd}_{2} \mathrm{Fe}_{14} \mathrm{~B}$ grain. The model was divided into $32 \times 32 \times 32$ or $64 \times 64 \times 64$ cubic elements, and the element size was either 1.5 or $3.0 \mathrm{~nm}$. Periodic boundary conditions were applied in all three directions, and consequently, an infinitely large magnet was assumed. The assumed physical parameters are shown in Table I.
Previously, Rave et al. studied the suitable element size for micromagnetic simulation of the switching field at the corner of a grain in which a magnetostatic field plays an important role. They reported that an element size equal to or smaller than the magnetostatic exchange length $\sqrt{2 A /\left(\mu_{0} J_{s}^{2}\right)}$ ( $A$ : exchange stiffness constant, $J_{\mathrm{s}}$ : saturation polarization, and $\mu_{0}$ : permeability of vacuum) is sufficient to obtain a correct switching field value. ${ }^{19}$ As the magnetostatic exchange length for our model magnet is approximately $2.9 \mathrm{~nm}$, the element size used in our simulation is equal to or smaller than the exchange length. Therefore, our element size should be sufficiently small. In fact, the calculation results using element sizes of 1.5 and $3.0 \mathrm{~nm}$ for $L=96 \mathrm{~nm}$ roughly agreed with each other.

The total magnetic energy $W$ stored in the model magnet, when an external field $H$ is applied, is given by

$$
W=K_{u}^{(H)} V \sum_{i=1}^{N^{3}}\left\{\frac{K_{u i}}{K_{u}^{(H)}}\left(\mathbf{u}_{\mathbf{i}} \cdot \mathbf{m}_{\mathbf{i}}\right)^{2}-\sum_{j=1}^{6}\left[\frac{J_{e i j} S}{6 K_{u}^{(H)} V}\left(\mathbf{m}_{\mathbf{i}} \cdot \mathbf{m}_{\mathbf{j}}\right)\right]-\frac{M_{s i}}{K_{u}^{(H)}}\left(\mathbf{m}_{\mathbf{i}} \cdot \mathbf{H}\right)\right\}+W_{m},
$$

where $K_{\mathrm{ui}}$ and $M_{s i}$ are the anisotropy constant and the saturation magnetization of the $i$ th element, respectively. $W_{m}$ represents the magnetostatic energy due to a local demagnetizing field. $N$ is the total number of elements, whereas $S$ and $V$ are the surface area and volume of each element, respectively. Furthermore, $\boldsymbol{u}_{\mathrm{i}}$ is a unit vector along the easy axis of the $i$ th element. $\boldsymbol{m}_{\mathrm{i}}$ and $\boldsymbol{m}_{\mathrm{j}}$ are the magnetization vectors in the $i$ th and the $j$ th elements, respectively, and are reduced by the saturation magnetization. Moreover, $J_{i j}$ is the exchange interaction constant per unit interface area between the $i$ th and $j$ th elements. The calculation method was described in detail elsewhere. ${ }^{20}$

\section{SIMULATION RESULTS AND DISCUSSION}

\section{A. Effect of non-magnetic phase size}

At first, the demagnetization process was simulated while varying the size $T$ of the non-magnetic phase from 0 to $72 \mathrm{~nm}$. In this calculation, the model size $L$ was $96 \mathrm{~nm}$, and the magnetic anisotropy constant $K_{\text {ud }}$ for the degraded surface layer was $K_{\mathrm{u}} / 2$. Figure 2 shows demagnetization curves for $T=6,30$, and $72 \mathrm{~nm}$. The volume fractions of the nonmagnetic phase for these $T$ value are $0.004,0.51$, and $7 \%$, respectively. The reverse domain was pinned in the degraded surface layer for $T=72 \mathrm{~nm}$, while magnetization reversal occurred immediately for $T=6$ and $30 \mathrm{~nm}$.

The calculated coercivity $h_{c}$ and the nucleation field $h_{\mathrm{n}}$ for the above model are shown in Fig. 3 as a function of $T$. The figure also shows the maximum demagnetizing field $h_{\mathrm{d}}$ present in the $\mathrm{Nd}_{2} \mathrm{Fe}_{14} \mathrm{~B}$ grain in the remanent state. $h_{c}, h_{\mathrm{n}}$, and $h_{\mathrm{d}}$ were reduced by the anisotropy field of $\mathrm{Nd}_{2} \mathrm{Fe}_{14} \mathrm{~B}$ $\left(=2 K_{\mathrm{u}} / J_{\mathrm{s}}=5.6 \mathrm{MA} / \mathrm{m}\right)$. Although $h_{\mathrm{d}}$ increased monotonically with increasing $T$, the behavior of $h_{c}$ was classified into three regions, regions I, II, and III. In region I $(20 \mathrm{~nm} \geq T)$, $h_{\mathrm{c}}$ was nearly constant. In region II $(20 \mathrm{~nm}<T<40 \mathrm{~nm}), h_{\mathrm{c}}$ decreased with increasing $T$ and agreed with $h_{\mathrm{n}}$. In region III ( $T \geq 40 \mathrm{~nm}$ ), $h_{\mathrm{c}}$ was determined by the de-pinning field and was constant. This behavior can be attributed to an effect of the demagnetizing field, because additional calculations neglecting the demagnetizing field resulted in a constant $h_{\mathrm{c}}$ value of approximately 0.61 , independent of $T$.

In region I, increasing $T$ did not affect $h_{\mathrm{c}}$ despite the resulting increase in the demagnetizing field. In order to understand this phenomenon more clearly, one-dimensional calculations were carried out for the model shown in the inset of Fig. 4. The demagnetization field $h_{\mathrm{d}}$ was localized in a region of width $W$, and a flat external field $h_{\text {ex }}$ was applied

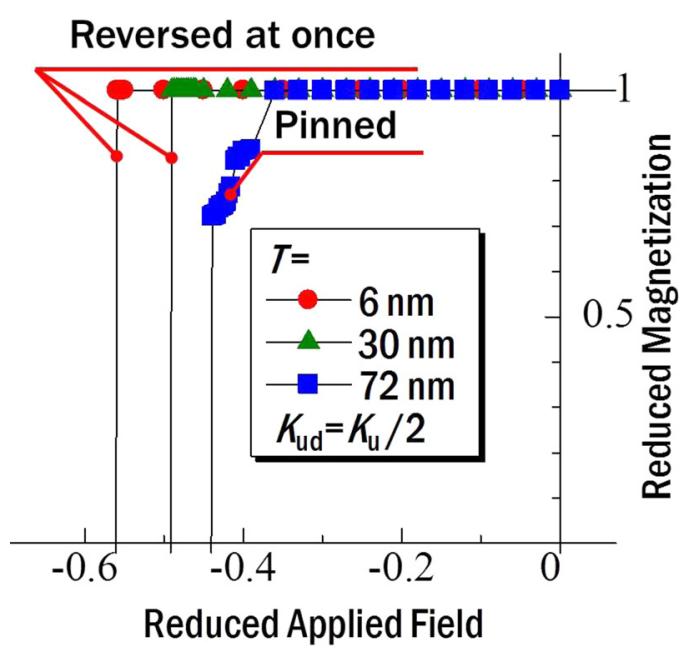

FIG. 2. Demagnetization curves for $L=96 \mathrm{~nm}$ and $T=6,30$, and $72 \mathrm{~nm}$. The magnetization and applied field were reduced by the saturation magnetization and the anisotropy field of the $\mathrm{Nd}_{2} \mathrm{Fe}_{14} \mathrm{~B}$, respectively. 


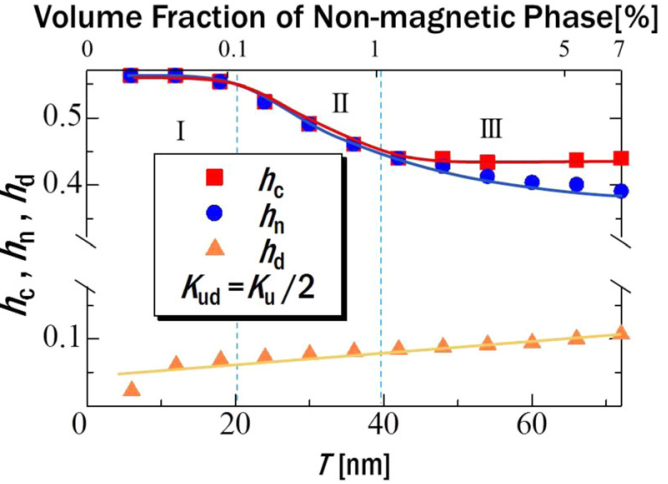

FIG. 3. Coercivity $h_{\mathrm{c}}$, nucleation field $h_{\mathrm{n}}$, and the maximum demagnetizing field $h_{\mathrm{d}}$ in the $\mathrm{Nd}_{2} \mathrm{Fe}_{14} \mathrm{~B}$ grain in the remanent state as a function of $T$. The volume fraction of the non-magnetic phase is also shown in the figure. $h_{\mathrm{c}}, h_{\mathrm{n}}$, and $h_{\mathrm{d}}$ are reduced by the anisotropy field of the $\mathrm{Nd}_{2} \mathrm{Fe}_{14} \mathrm{~B}$ $\left(=2 K_{\mathrm{u}} / J_{\mathrm{s}}=5.6 \mathrm{MA} / \mathrm{m}\right)$.

opposite to the direction of magnetization. $h_{\mathrm{c}}$ was calculated by varying $h_{\mathrm{ex}}$ under fixed $W$ and $h_{\mathrm{d}}$ values. As seen in Fig. $4, h_{\mathrm{c}}$ was markedly affected when $W$ reached $10 \mathrm{~nm}$, suggesting that the demagnetizing field had a significant effect on $h_{\mathrm{c}}$ only when it exceeded a critical size. This critical size effect explains the constant $h_{\mathrm{c}}$ value in region I, as shown in Fig. 3.

\section{B. Effect of model size}

Figure 5 shows $h_{\mathrm{c}}$ as a function of $T / L$ for $L=48,96$, and $192 \mathrm{~nm}$. The thickness of the degraded surface layer $D$ was kept at $3 \mathrm{~nm}$, and $K_{\mathrm{ud}}$ was set to $K_{\mathrm{u}}$ and $K_{\mathrm{u}} / 2$ in the calculations shown in (a) and (b), respectively. Clearly, $h_{\mathrm{c}}$ increased with decreasing $L$. It should be noted that the magnitude of the demagnetizing field for a given $T / L$ value is expected to be nearly the same for all $L$ values, because the geometric configuration of the $\mathrm{Nd}_{2} \mathrm{Fe}_{14} \mathrm{~B}$ grain, nonmagnetic phase, and grain boundaries are unaffected by the $L$ value, and the demagnetization field is determined by their configuration rather than their size. For example, the demagnetizing field coefficient does not depend on the magnet size.

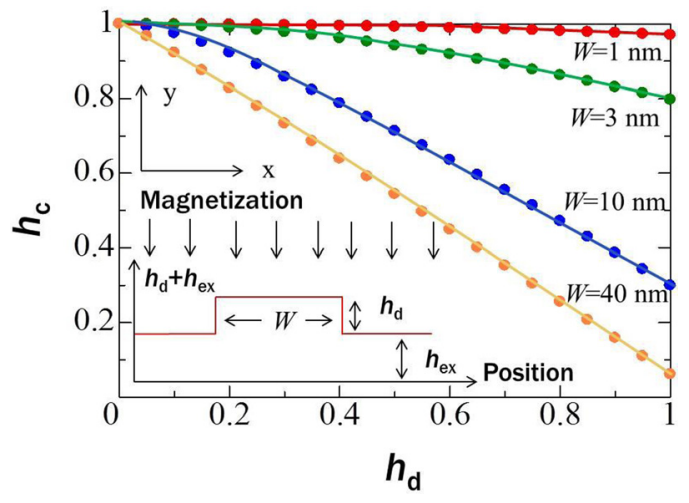

FIG. 4. Coercivity $h_{\mathrm{c}}$, as obtained by one-dimensional calculations in which the direction of magnetization was assumed to be a function of $x$. The easy axis of magnetization lies in the $\pm y$ direction, and the magnetization was assumed to initially be in the $-y$ direction. The demagnetization field $h_{\mathrm{d}}$ localized in the region with width $W$ and under a flat external field $h_{\mathrm{ex}}$ were applied in the direction opposite to the magnetization (the $+y$ direction). $h_{\mathrm{c}}, h_{\mathrm{d}}$, and $h_{\mathrm{ex}}$ were reduced by the anisotropy field of the $\mathrm{Nd}_{2} \mathrm{Fe}_{14} \mathrm{~B}\left(=2 K_{\mathrm{u}} / J_{\mathrm{s}}=5.6 \mathrm{MA} / \mathrm{m}\right)$.

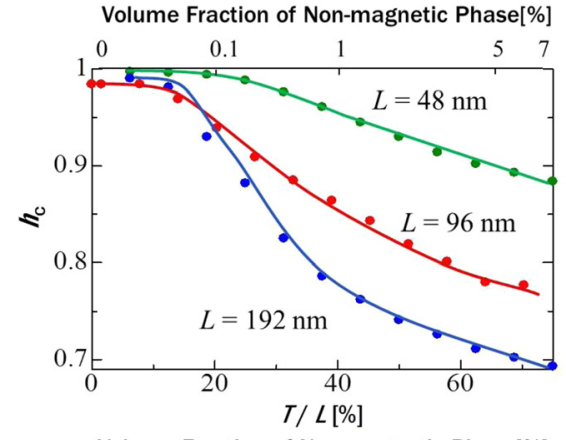

(a) $K_{\mathrm{ud}}=K_{\mathrm{u}}$

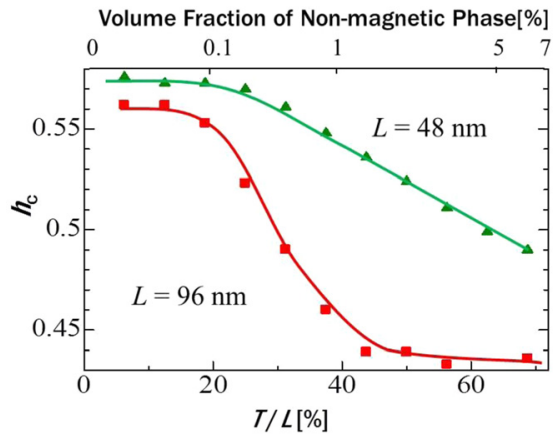

(b) $K_{\mathrm{ud}}=K_{\mathrm{u}} / 2$

FIG. 5. Coercivity $h_{\mathrm{c}}$ for $L=48,96$, and $192 \mathrm{~nm}$ as a function $T / L$. The magnetic anisotropy constant $K_{\mathrm{ud}}$ in the surface layers of the $\mathrm{Nd}_{2} \mathrm{Fe}_{14} \mathrm{~B}$ was set to $K_{\mathrm{u}}$ and $K_{\mathrm{u}} / 2$ in (a) and (b), respectively. $h_{\mathrm{c}}$ was reduced by the anisotropy field of the $\mathrm{Nd}_{2} \mathrm{Fe}_{14} \mathrm{~B}\left(=2 K_{\mathrm{u}} / J_{\mathrm{s}}=5.6 \mathrm{MA} / \mathrm{m}\right)$.

Examples of demagnetizing field distributions in the remanent state are shown in Fig. 6 for $K_{\mathrm{ud}}=K_{\mathrm{u}}$ and $T / L=0.5$. The demagnetizing field was calculated at a position $L / 64$ away from the surfaces of the $\mathrm{Nd}_{2} \mathrm{Fe}_{14} \mathrm{~B}$ grain, as shown schematically in the inset. The distances from the surfaces, $L / 64$, correspond to $1.5 \mathrm{~nm}$ for $L=96 \mathrm{~nm}$ and $0.75 \mathrm{~nm}$ for $L=48 \mathrm{~nm}$. The half-width of the $h_{\mathrm{d}}$ vs. position curve for $L=48 \mathrm{~nm}$ is $1 / 2$ that for $L=96 \mathrm{~nm}$, whereas the maximum value of $h_{\mathrm{d}}$ for $L=48 \mathrm{~nm}$ is the same as that for $L=96 \mathrm{~nm}$. Therefore, the high $h_{\mathrm{c}}$ values for $L=48 \mathrm{~nm}$ are consistent because the results shown in Fig. 4 indicate that a narrow distribution of $h_{\mathrm{d}}$ results in a high $h_{\mathrm{c}}$ value for a given $h_{\mathrm{d}}$ value (a given $T / L$ value). It should be noted that the increase in $h_{\mathrm{c}}$ due to the reduction in $L$ can be attributed to a decrease in the size of the non-magnetic phase, which varies

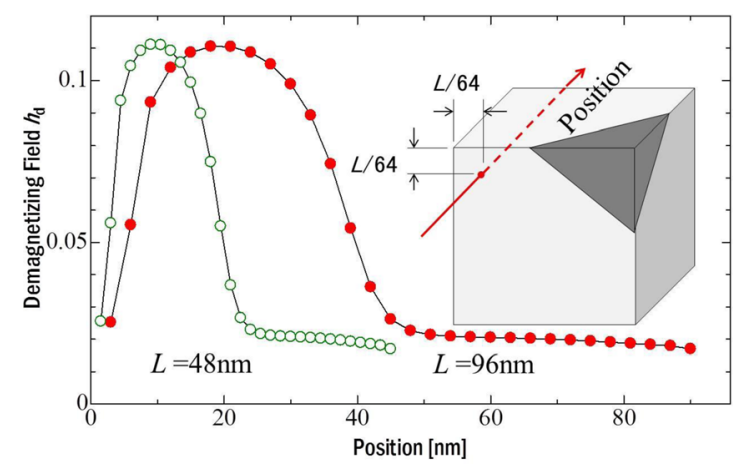

FIG. 6. Examples of demagnetizing field $h_{\mathrm{d}}$ distributions in the remanent state for $K_{\mathrm{ud}}=K_{\mathrm{u}}$ and $T / L=0.5$. The calculation position is $L / 64$ away from the surfaces of the $\mathrm{Nd}_{2} \mathrm{Fe}_{14} \mathrm{~B}$ grain, as shown in the inset. The distances from the surfaces correspond to $1.5 \mathrm{~nm}$ for $L=96 \mathrm{~nm}$ and $0.75 \mathrm{~nm}$ for $L=48 \mathrm{~nm}$. $h_{\mathrm{d}}$ was reduced by the anisotropy field of the $\mathrm{Nd}_{2} \mathrm{Fe}_{14} \mathrm{~B}$ $\left(=2 K_{\mathrm{u}} / J_{\mathrm{s}}=5.6 \mathrm{MA} / \mathrm{m}\right)$. 
proportionally with $L$. This reduction in the size of the nonmagnetic phase may be one origin of the high $h_{\mathrm{c}}$ values that were obtained experimentally for magnets with a small grain size, because refinement of the $\mathrm{Nd}_{2} \mathrm{Fe}_{14} \mathrm{~B}$ grains should accompany the refinement of the non-magnetic phases.

It has already been reported on the basis of micromagnetic simulations that a decrease in grain size increases $h_{\mathrm{c}}$ due to a decrease in the demagnetizing field. ${ }^{15}$ This result is not inconsistent with our result because of the difference in the calculation models employed. In the calculations reported in Ref. 15, the grains nearest to the surface of the magnet were assumed to be magnetically soft, and the reversed magnetization in these grains became the source of the demagnetizing field. Consequently, the volume fraction of these grains decreased with decreasing grain size. Therefore, the decrease in demagnetization field can be attributed to the decrease in the volume fraction of magnetically soft grains. In our model, on the other hand, the configuration of the $\mathrm{Nd}_{2} \mathrm{Fe}_{14} \mathrm{~B}$ grain, the non-magnetic phase, and the grain boundaries was the same for $L=48,96$, and $192 \mathrm{~nm}$. As a result, the value of $h_{\mathrm{d}}$ was not affected by $L$. Therefore, our simulation results suggest that a narrow distribution of $h_{\mathrm{d}}$ may be another origin of the experimentally observed increase in $h_{\mathrm{c}}$ due to refinement of the microstructure.

\section{CONCLUSIONS}

The effect of the presence of a non-magnetic phase at multi-junctions of $\mathrm{Nd}_{2} \mathrm{Fe}_{14} \mathrm{~B}$ grains on the demagnetization of Nd-Fe-B magnets was studied by micromagnetic simulations. The main findings are summarized as follows:

(1) The non-magnetic phase created a strong local demagnetizing field and assisted the magnetization reversal. The effect of the demagnetizing field was only significant when the size of the non-magnetic phase exceeded a critical value.
(2) The critical size effect can be explained by a change in the spatial distribution of the demagnetizing field.

(3) Microstructural refinement increased the coercivity through a change in the spatial distribution of the demagnetizing field, as mentioned above. The critical size effect represents another origin of the experimentally observed increase in coercivity due to refinement of the microstructure.

${ }^{1}$ S. Sugimoto, J. Jpn. Soc. Powder Powder Metall. 57, 395 (2010).

${ }^{2}$ A. Sakuma, S. Takeda, and M. Endoh, IEEE Trans. J. Magn. Jpn. 9, 127 (1994).

${ }^{3}$ H. Fukunaga and T. Fukuda, Jpn. J. Appl. Phys. 29, 1711 (1990).

${ }^{4}$ G. Hrkac, T. G. Woodcock, C. Freeman, A. Goncharov, J. Dean, T. Schrefl, and O. Gutfleisch, Appl. Phys. Lett. 97, 232511 (2010).

${ }^{5}$ S. Tanaka, H. Moriya, H. Tsuchiura, A. Sakuma, M. Divis, and P. Novak, J. Appl. Phys. 109, 07A702 (2011).

${ }^{6}$ H. Sepehri-Amin, T. Ohkubo, T. Shima, and K. Hono, Acta Mater. 60, 819 (2012).

${ }^{7}$ H. Kronmüller and K. D. Durst, J. Magn. Magn. Mater. 74, 291 (1988).

${ }^{8}$ Y. Une and M. Sagawa, J. Jpn. Inst. Metals 76, 12 (2012).

${ }^{9}$ P. Yi, M. Lin, R. Chen, D. Lee, and A. Yan, J. Alloys Compd. 491, 605-609 (2010).

${ }^{10}$ T. Schrefl, H. F. Schmidts, J. Fidler, and H. Kronmüller, J. Appl. Phys. 73, 6510 (1993)

${ }^{11}$ D. Süss, T. Schrefl, and J. Fidler, IEEE Trans. Magn. 36, 3282 (2000).

${ }^{12}$ J. Liu, H. Sepehri-Amin, T. Ohkubo, K. Hioki, A. Hattori, T. Schrefl, and K. Hono, Acta Mater. 61, 5387 (2013).

${ }^{13}$ J. Fidler and T. Schrefl, J. Appl. Phys. 79, 5029 (1996).

${ }^{14}$ T. Schrefl, H. F. Schmidts, J. Fidler, and H. Kronmüller, IEEE Trans. Magn. 29, 2878 (1993).

${ }^{15}$ H. F. Schmidts and H. Kronmüller, J. Magn. Magn. Mater. 94, 220 (1991).

${ }^{16}$ H. Sepehri-Amin, T. Ohkubo, M. Gruber, T. Schrefl, and K. Hono, Scr. Mater. 89, 29 (2014).

${ }^{17}$ S. Bance, H. Oezelt, A. T. Schrefl, G. Ciuta, N. M. Dempsey, D. Givord, M. Winklhofer, G. Hrkac, G. Zimanyi, O. Gutfleisch, T. G. Woodcock, T. Shoji, M. Yano, A. Kato, and A. Manabe, Appl. Phys. Lett. 104, 182408 (2014).

${ }^{18}$ H. Fukunaga, K. Kirino, M. Nakano, and T. Yanai, J. Jpn. Inst. Metals 76, 43 (2012).

${ }^{19}$ W. Rave, K. Ramsto, and A. Hubert, J. Magn. Magn. Mater. 183, 329 (1998).

${ }^{20}$ H. Fukunaga, J. Kuma, and Y. Kanai, IEEE Trans. Magn. 35, 3235 (1999). 